\title{
ON THE METHOD OF MONITORING OF CLASSICAL VIBRATIONS IN SODIUM DIMER*
}

\author{
P. KowalCzyK AND C. RADzewicz \\ Institute of Experimental Physics, Warsaw University, Hoża 69, 00-681 Warszawa, \\ Poland
}

(Received March 6, 1990; in revised form May 15, 1990)

\begin{abstract}
A computer simulation of an experiment allowing for monitoring of vibrational motion of the wave packet in the $\mathrm{Na}_{2}$ molecule is presented. The experiment is based on pump and delayed probe excitation followed by observation of laser-induced fluorescence.
\end{abstract}

PACS numbers: $33.90 .+\mathrm{h}$

\section{Introduction}

A basic difference between macro- and micro-objects is in a density of their energy levels. The levels of a typical clock pendulum swinging with a frequency of $1 \mathrm{~Hz}$ are separated by approximately $10^{-10} \mathrm{~cm}^{-1}$. No amount of mechanical energy can be transferred to the pendulum with such an accuracy and there is no certainty which particular energy level is occupied by the system. In consequence, the pendulum should be described by a superposition of a great many eigenfunctions and their interference produces a wave packet. It is well localized in space and its centre moves classically, according to our everyday experience.

The energy levels of micro-objects are located much more closely. For example, vibrational levels in most electronic states of the thoroughly studied $\mathrm{Na}_{2}$ molecule are separated by about $100 \mathrm{~cm}^{-1}$. This facilitates excitation of a well-defined molecular eigenstate which can be described quantum-mechanically by a single wave function. However, a recent availability of ultra-short optical pulses has changed the experimental possibilities. The finite duration $\tau$ of a femtosecond laser pulse produces a frequency bandwidth which corresponds to a spectral width

*This research was supported by the Polish Ministry of National Education under the research projects CPBP 01.06 and RPBR RRI.14. 
of $\Delta \omega \approx 2 \pi / \tau$, being of the order of several hundred $\mathrm{cm}^{-1}$. When such a pulse is used to excite a molecule to the excited electronic state, many vibrational levels will be simultaneously and coherently excited. This imposes a classical behaviour upon the molecule, corresponding to the motion of two masses connected by a spring. If the spatial distribution of the vibrational wave function at the end of the pulse is calculated, it is found that the short pulse has excited a well-defined wave packet, concentrated in a relatively small range of internuclear distances $R$. This wave packet evolves following the classical trajectory limited in space by the classical turning points.

In recent years the spatially localized wave packets have been investigated in two types of quantum systems. In Rydberg atoms coherent superpositions of atomic states were excited, resulting in wave packets localized either in radial or angular coordinates [1-5]. The fundamental concern of these studies was to understand the transition between classical and quantum physics. However, practical application of the Rydberg atom wave packets seems to be questionable. The other system of concern were molecules and vibrational wave packets, already mentioned above $[6,7$ and references therein]. In this case the interest in molecular states with well defined $R$ was motivated by their potential applications in laser chemistry. Several processes as electronic excitation, photo-dissociation or collisional redistribution, are known to depend strongly on internuclear distance and the ability to control the $R$ value in a molecule may provide a way of enhancing a desirable process out of several competing ones.

In this study we present a computer simulation of an experiment allowing one to monitor vibrational motion of the wave packet created in the $\mathrm{Na}_{2}$ molecule. The wave packet is excited by the femtosecond laser pulse and probed by a second ultra-short pulse which further excites the molecule. Both pulses originate from the same laser which provides all practical advantages of one-colour experiment. The intensity of the subsequent laser-induced fluorescence depends on time delay between two pulses and reflects time evolution of the wave packet.

The preliminary analysis [8] dealt with a two-colour experiment and suggested another way to monitor the wave packet motion. Additionally, it was partially based on approximate theoretical potential curves for the $\mathrm{Na}_{2}$ molecule. The present work employs solely the accurate potentials determined experimentally. An analysis of conditions required for the future experiment is also given.

\section{The model experiment}

We assume the following conditions for the model experiment. Let us consider a crossed laser beam - molecular beam experiment. The dye laser with $630 \mathrm{~nm}$ central wavelength produces Fourier limited Gaussian pulses of $50 \mathrm{fs}$ duration (spectral width of about $600 \mathrm{~cm}^{-1}$ ) and repetition rate less than $30 \mathrm{MHz}$. The train of pulses is split by a $50 \%$ beam splitter into a pump beam and suitably delayed probe beam. The pump beam excites $\mathrm{Na}_{2}$ molecules from the $v^{\prime \prime}=0$ vibrational level in the ground $\mathrm{X}^{1} \Sigma_{\mathrm{g}}^{+}$state to several vibrational levels in the $\mathrm{A}^{1} \Sigma_{\mathrm{u}}^{+}$state centred around $v^{\prime} \approx 10$ and creates a wave packet oscillating between 
the classical turning points in this state. The probe beam transfers the excitation to the $(2)^{1} \Pi_{g}$ Rydberg state with efficiency depending on the spatial position of the wave packet. Excitation of other molecular Rydberg states by the probe pulse is highly non-resonant and will be neglected in further analysis. Population of the $(2)^{1} \Pi_{\mathrm{g}}$ state is monitored by observation of the spontaneous fluorescence $(2)^{1} \Pi_{\mathrm{g}} \rightarrow$ $\mathrm{B}^{1} \Pi_{u}$, perpendicularly to the laser and molecular beams. The fluorescence light of this particular transition has the wavelength in the range of 790-1400 nm and is selected by a long-pass filter opening at about $800 \mathrm{~nm}$. The filter blocks both the $(2)^{1} \Pi_{\mathrm{g}} \rightarrow \mathrm{A}^{1} \Sigma_{\mathrm{u}}^{+}$and the $\mathrm{A}^{1} \Sigma_{\mathrm{u}}^{+} \rightarrow \mathrm{X}^{1} \Sigma_{\mathrm{g}}^{+}$fluorescence centred around the laser wavelength as well as any atomic fluorescence excited by spectral wings of the laser line. The fluorescence signal transmitted by the filter is detected by a photomultiplier and recorded as a function of the delay time between the pump and probe pulses.

\section{Method of calculations}

In a pump-probe pulse experiment considered here the duration of the pump pulse is shorter than the vibrational period. Therefore it creates a non-stationary wave packet and the probe pulse measures the optical absorption of the non-stationary system. The electric field of the laser pulses is written in the form:

$$
\boldsymbol{E}_{i}(t)=E_{0 i}(t) e \exp (i \omega t)+c . c .,
$$

with $i=1,2$ denoting the exciting and probe pulses, respectively. $E_{0 i}(t)$ are the pulse envelopes, both assumed to be Gaussian with the same width. The pump pulse is peaked at $t=0$ and the probe pulse at $t=t_{0}$. The symbol $e$ denotes the polarization vector of the electric field and $\omega$ is the angular frequency.

In the presently considered experimental arrangement $\mathrm{Na}_{2}$ molecules are prepared in the lowest vibrational level $\mid 0>$ of the ground $\mathrm{X}^{1} \Sigma_{\mathrm{g}}^{+}$state. We set the energy of this level to zero in the energy scale.

We find the time-dependent wavefunction $\mid \Psi_{\mathrm{A}}(t)>$ of the molecule in the intermediate $\mathrm{A}^{1} \Sigma_{u}^{+}$state after the excitation using the perturbation theory approximation. It has to be taken into account that the time duration of the pump pulse is not negligible in comparison to the vibrational period. To deal with the problem numerically, we introduce the discrete variable $n \tau$, where $n$ is an integer and $\tau$ is a small time step. The function $\left|\Psi_{\mathrm{A}}(t)\right\rangle$ is a sum of amplitudes, each representing the state of the molecule at a time $t$ if the photon was absorbed at $n \tau$. The state $\left|\Psi_{\mathrm{A}}(t)\right\rangle$, being a sum of several wave packets, is often called a wave train [9] and is given by

$$
\left.\left|\Psi_{\mathrm{A}}(t)>=\sum_{n<t / \tau} \sum_{a} E_{01}(n \tau)\right| a\right\rangle\left\langle a\left|\mu_{\mathrm{AX}}\right| 0\right\rangle \exp \left[-\mathrm{i} \mathcal{E}_{a}(t-n \tau) / \hbar\right] .
$$

Here $\mu_{\mathrm{AX}}$ is the electric dipole transition moment for the $\mathrm{A}^{1} \Sigma_{\mathrm{u}}^{+} \leftarrow X^{1} \Sigma_{\mathrm{g}}^{+}$transition in $\mathrm{Na}_{2}$ and $\mid a>$ are the stationary wavefunctions of the vibrational levels with energies $\mathcal{E}_{a}$ in the intermediate $\mathrm{A}^{1} \Sigma_{u}^{+}$state. 
The second, probe pulse excites the $(2)^{1} \Pi_{\mathrm{g}} \leftarrow \mathrm{A}^{1} \Sigma_{\mathrm{u}}^{+}$transition and generates a wave train in the $(2)^{1} \Pi_{\mathrm{g}}$ state, which is, for $t>t_{0}$,

$$
\left|\Psi_{\mathrm{R}}(t)>=\sum_{r} A_{r}\right| r>\exp \left(-\mathrm{i} \mathcal{E}_{r} t / \hbar\right) .
$$

In the above $\mid r>$ and $\mathcal{E}_{r}$ are the wavefunctions and energies of vibrational levels in the $(2)^{1} \Pi_{\mathrm{g}}$ state and the amplitude $A_{r}$ of each $\mid r>$ level is given by

$$
\begin{array}{r}
A_{r}=\sum_{n<t / \tau} \sum_{n<k<t / \tau} \sum_{a} E_{02}(k \tau) E_{01}(n \tau)<r\left|\mu_{\mathrm{RA}}\right| a><a\left|\mu_{\mathrm{AX}}\right| 0> \\
\times \exp \left(\mathrm{i} \mathcal{E}_{m} n \tau / \hbar\right) \exp \left[\mathrm{i}\left(\mathcal{E}_{r}-\mathcal{E}_{m}\right) k \tau / \hbar\right] .
\end{array}
$$

The notation $\mu_{\mathrm{RA}}$ is used for the electric dipole transition moment for the $(2)^{1} \Pi_{\mathrm{g}} \leftarrow$ $\mathrm{A}^{1} \Sigma_{u}^{+}$transition. Since the envelope $E_{02}$ of the probe pulse is shifted corresponding to the envelope $E_{01}$ of the pump pulse, the amplitudes $A_{r}$ depend on the delay $t_{0}$ between both pulses.

If the $(2)^{1} \Pi_{g} \rightarrow B^{1} \Pi_{u}$ fluorescence is observed under conditions of time averaging by a typical photodetector with a nanosecond time response, the fluorescence intensity as a function of time delay between the pump and probe pulses is given by

$$
I\left(t_{0}\right) \propto \sum_{r, b}\left|A_{r}\left(t_{0}\right)\right|^{2}|<r| \mu_{\mathrm{BR}}|b>|^{2} .
$$

Here $\mid b>$ stands for vibrational wavefunctions in the $\mathrm{B}^{1} \Pi_{\mathrm{u}}$ state and $\mu_{\mathrm{BR}}$ is the electric dipole transition moment for the $(2)^{1} \Pi_{\mathrm{g}} \rightarrow \mathrm{B}^{1} \Pi_{\mathrm{u}}$ transition.

It must be taken into account that in addition to the subsequent absorption of the first photon from the pump beam and the second one from the probe beam, excitation of the $(2)^{1} \Pi_{\mathfrak{g}}$ state can be achieved by absorption of two pump photons or two probe photons. This is a consequence of deriving pump and probe beams from the same laser. On the other hand, because of the short lifetime of the $\mathrm{A}^{1} \Sigma_{u}^{+}$ state (12 ns [10]) and the assumed laser repetition rate less than $30 \mathrm{MHz}$, at the arrival of a given pump pulse we can neglect population of the A state by all previous pulses.

To calculate $I\left(t_{0}\right)$ one has to know the stationary vibrational wavefunctions $|0>| a>,, \mid r>$ and $\mid b>$. They were obtained by solving the appropriate radial Schrödinger equations numerically, using the method described by Cooley [11]. Potential energy curves for the states $\mathrm{X}^{1} \Sigma_{\mathrm{g}}^{+}, \mathrm{A}^{1} \Sigma_{\mathrm{u}}^{+}, \mathrm{B}^{1} \Pi_{\mathrm{u}}$ and $(2)^{1} \Pi_{\mathrm{g}}$ were taken from Refs. [12-16]. We assumed that $\mu_{\mathrm{XA}}, \mu_{\mathrm{AR}}$ and $\mu_{\mathrm{RB}}$ do not depend substantially on the internuclear distance, what is a reasonable approximation for the limited range of $R$ sampled by the wave packets.

\section{Discussion}

Figure 1 displays intensity of the total $(2)^{1} \Pi_{\mathrm{g}} \rightarrow \mathrm{B}^{1} \Pi_{\mathrm{u}}$ fluorescence $I$ versus time delay $t_{0}$ between the pump and probe pulses. The uniform background visible 


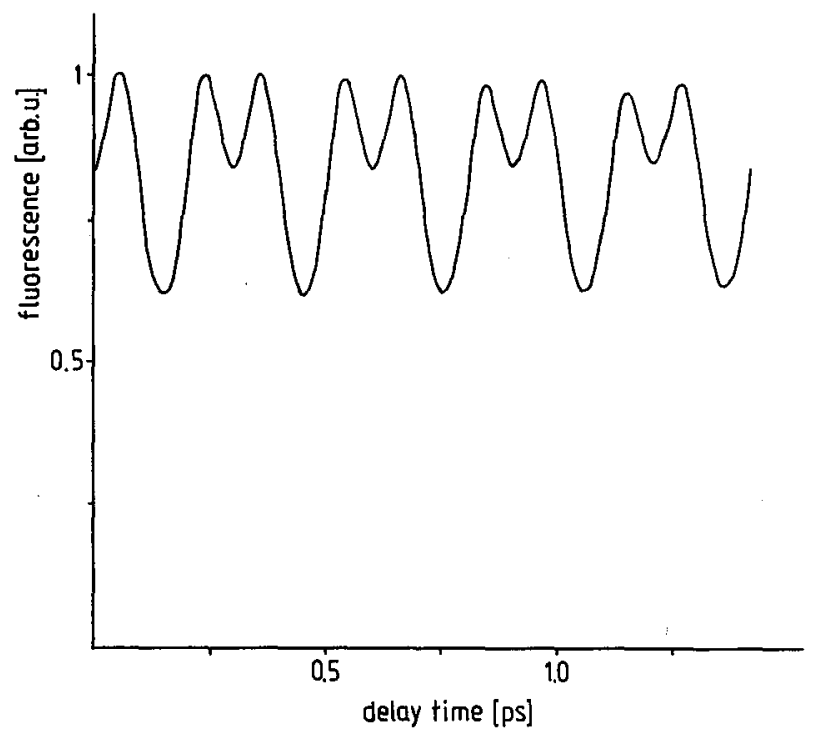

Fig. 1. (2) $)^{1} \Pi_{g} \rightarrow B^{1} \Pi_{u}$ fluorescence intensity versus the delay time between the pump and probe pulses.

in the figure originates from simultaneous absorption of two pump or two probe photons. It can be cancelled out when the pump and probe beams are chopped with different frequencies $\Omega_{1}$ and $\Omega_{2}$ and the fluorescence signal is measured with a lock-in technique on the frequency $\Omega_{1} \pm \Omega_{2}$.

The fluorescence signal is proportional to the absorption of the probe pulses by the wave train generated in the A state. Therefore, the $I(t)$ dependence reflects spatial evolution of this wave train. Absorption of the probe photon at $t=0$ occurs at the place, where the A state wave train has been created. This is around the equilibrium distance of the ground $\mathrm{X}^{1} \Sigma_{\mathrm{g}}^{+}$state $\left(R_{e} \approx 3.08 \AA\right.$ [12]), being simultaneously the inner turning point for the oscillatory motion of the wave train. As one can see from Fig. 1 the probability of the $(2)^{1} \Pi_{\mathrm{g}} \leftarrow \mathrm{A}^{1} \Sigma_{\mathrm{u}}^{+}$absorption is, in this case, moderate. The probability increases when the wave train moves towards greater $R$ values and reaches maximum for $t=60$ fs (equivalent to $R=3.52 \AA$ ). Then it decreases to the minimum value at the outer turning point of the wave train motion $(t=T / 2=155 \mathrm{fs})$. Afterwards the wave train travels back passing again through the $R$ value of maximum absorption probability and returns to its starting point being the inner turning point.

The numerically predicted dependence of the $(2)^{1} \Pi_{\mathrm{g}} \leftarrow \mathrm{A}^{1} \Sigma_{\mathrm{u}}^{+}$absorption on the spatial position of the A state wave train can be explained qualitatively in a semiclassical way. The Franck-Condon principle requires conservation of both position and momentum (or kinetic energy) of the nuclei during the electronic transition. If the transition starts from a given $\mathcal{E}^{\prime}$ level, the final points which clas- 
sically conserve nuclear position and momentum lie on the Mulliken potential [17]:

$$
X(R)=U(R)+\mathcal{E}^{\prime}-U^{\prime}(R),
$$

where $U^{\prime}(R)$ and $U(R)$ represent the potential curves of the initial and final states, respectively. In Fig. 2 the loci of Mulliken potentials are shown for transitions from vibrational levels in the $\mathrm{A}^{1} \Sigma_{\mathrm{u}}^{+}$state predominantly populated by the pump pulse to the $(2)^{1} \Pi_{\mathrm{g}}$ state. It is to be remembered that due to the uncertainty principle the wave train energy is spread around the $v^{\prime}=10$ energy level in the A state and the probe photon energy is spread around $\hbar \omega_{0}$. Still it can be seen from Fig. 2 that

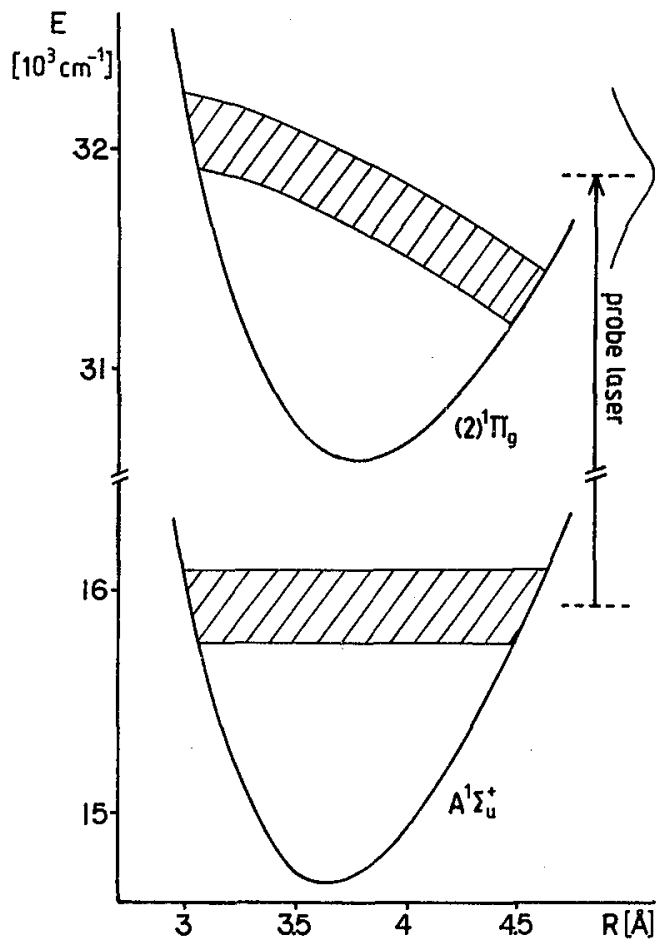

Fig. 2. Potential curves for the $\mathrm{A}^{1} \Sigma_{\mathrm{u}}^{+}$and $(2)^{1} \Pi_{\mathrm{g}}$ states in $\mathrm{Na}_{2}$ molecule. The dashed area in the lower potential indicates energy spread of the wave train excited by the pump pulse. The dashed area in the upper potential corresponds to loci of Mulliken potentials for transitions starting from the energy range indicated in the lower potential. The energy of the probe photon as well as its spread are also shown.

the position and momentum conservation are best fulfilled at about $R \approx 3.5 \AA$, less precisely at the inner turning point and very poorly at the outer turning point.

In order to evaluate the feasibility of our model experiment, we have estimated the intensity of the $(2)^{1} \Pi_{\mathrm{g}} \rightarrow \mathrm{B}^{1} \Pi_{\mathrm{u}}$ fluorescence. The following assumptions were made: laser pulse energy is $2 \times 10^{-10} \mathrm{~J}$, the density of $\mathrm{Na}_{2}$ in the supersonic 
molecular beam equals $10^{14} \mathrm{~cm}^{-3}$ and the cylindrical interaction zone is $100 \mu \mathrm{m}$ in diameter and $2 \mathrm{~mm}$ long.

The probability $P$ of excitation of a $\mathrm{Na}_{2}$ molecule from the ground $\mathrm{X}^{1} \Sigma_{\mathrm{g}}^{+}$state to the $\mathrm{A}^{1} \Sigma_{\mathrm{u}}^{+}$state per single laser pulse was calculated using the laser parameters and Einstein $A$ coefficients for this transition [18]. The resulting probability $P \approx 2 \times 10^{-4}$ justifies the perturbation method applied in the calculations. It is reasonable to assume that the transition probability for the $(2)^{1} \Pi_{\mathrm{g}} \leftarrow \mathrm{A}^{1} \Pi_{\mathrm{u}}$ is comparable to that for the $\mathrm{A} \leftarrow \mathrm{X}$ transition. Therefore, the probability for a molecule to be excited by two subsequent pulses to the $(2)^{1} \Pi_{\mathrm{g}}$ state is approximately $4 \times 10^{-8}$. Since there are about $10^{9}$ molecules in the excitation region, we can expect more than one molecule being transferred to the upper state at every laser pulse. We have estimated that the $(2)^{1} \Pi_{g} \rightarrow B^{1} \Pi_{u}$ fluorescence is a dominating channel for decay of the upper state population; thus the number of fluorescence photons is roughly the same as the number of excited molecules. Taking into account laser repetition rate we arrive at conclusion that there are $10^{7}$ to $10^{8}$ photons per second in the measured fluorescence which should be enough for a measurement with a standard lock-in technique.

Finally we compare properties of a vibrational wave packet in molecule and the Rydberg atom wave packet. A time duration of exciting laser pulses required in both cases is substantially different. Close packing of atomic Rydberg states in energy scale $\left(\Delta \mathcal{E} \approx 0.01 \mathrm{~cm}^{-1}\right.$ for $\left.n \approx 50\right)$ allows one to use relatively long laser pulses up to $500 \mathrm{ps}$ in duration [4]. In the model $\mathrm{Na}_{2}$ molecule vibrational levels are separated by about $100 \mathrm{~cm}^{-1}$ and pulses of few tens of femtoseconds are necessary. On the other hand, excitation of molecular wave packets shows some advantages. Due to a uniform energy spacing of vibrational levels (imposed by approximately harmonic potential) a molecular wave packet remains localized in space during many periods of vibrations, whereas the Rydberg atom wave packet, particularly when localized in radial coordinate, disperses after few oscillations [1]. Moreover, excitation of the atomic wave packet, especially formed of the high angular momentum Rydberg states, presents serious experimental difficulties and requires optical excitation of an atom additionally dressed by an of field $[3,4]$. Excitation of the vibrational wave packet in a molecule is experimentally easier and relatively simple for theoretical analysis.

\section{References}

[1] J. Parker, C.R. Stroud Jr., Phys. Rev. Lett. 56, 716 (1986).

[2] G. Alber, H. Ritsch, P. Zoller, Phys. Rev. A 34, 1058 (1986).

[3] J.A. Yeazell, C.R. Stroud Jr., Phys. Rev. A 35, 2806 (1987).

[4] J.A. Yeazell, C.R. Stroud Jr., Phys. Rev. Lell. 60, 1494 (1988).

[5] G. Alber, T. Haslwanter, P. Zoller, J. Opt. Soc. Am. B, Opt. Phys. 5, 2439 (1988).

[6] A.H. Zewail, Science 242, 1645 (1988).

[7] A.H. Zewail, R. Bernstein, Chem. Eng. News 66, 24 (1988). 
[8] C. Radzewicz, M.G. Raymer, in: Atomic and Molecular Processes with Short Intense Laser Pulses, ed. A. Bandrauk, Plenum, New York 1988, p. 107.

[9] V. Engel, H. Metin, R. Almeida, R.A. Marcus, A.H. Zewail, Chem. Phys. Lett. 152, 1 (1988).

[10] T.W. Ducas, M.G. Littman, M.L. Zimmerman, D. Kleppner, J. Chem. Phys. 65, 842 (1976).

[11] J.H. Cooley, Math. Comput. 15, 363 (1961).

[12] P. Kusch, M.M. Hessel, J. Chem. Phys. 68, 2591 (1978).

[13] K.K. Verma, J.T. Bahns, A.R. Rajaei-Rizi, W.C. Stwalley, W.T. Zemke, J. Chem. Phys. 78, 3599 (1983).

[14] R.F. Barrow, J. Verges, C. Effantin, K. Hussein, J. d'Incan, Chem. Phys. Lett. 104, 179 (1984).

[15] G. Gerber, R. Möller, Chem. Phys. Lett. 113, 546 (1985).

[16] A.J. Taylor, K.M. Jones, A.L. Schawlow, J. Opt. Soc. Am. 73, 994 (1983).

[17] R.S. Mulliken, J. Chem. Phys. 55, 309 (1971).

[18] W.J. Stevens, M.M. Hessel, P.J. Bertoncini, A.C. Wahl, J. Chem. Phys. 66, 1477 (1977). 